\title{
Finding Rare Disease Patients in EHR Databases via Lightly-Supervised Learning
}

\author{
Rich Colbaugh Kristin Glass \\ Volv Global \\ Lausanne, Switzerland
}

\begin{abstract}
There is considerable interest in developing computational models capable of detecting rare disease patients in population-scale databases such as electronic health records (EHRs). Deriving these models is challenging for several reasons, perhaps the most daunting being the limited number of already-diagnosed, 'labeled' patients from which to learn. We overcome this obstacle with a novel lightly-supervised algorithm that leverages unlabeled and/or unreliably-labeled patient data - which is typically plentiful - to facilitate model induction. Importantly, we prove the algorithm is safe: adding unlabeled/unreliably-labeled data to the learning procedure produces models which are usually more accurate, and guaranteed never to be less accurate, than models learned from reliably-labeled data alone. The proposed method is shown to substantially outperform state-of-the-art models in patient-finding experiments involving two different rare diseases and a country-scale EHR database. Additionally, we demonstrate feasibility of transforming high-performance models generated through light supervision into simpler models which, while still accurate, are readily-interpretable by non-experts.
\end{abstract}

\section{Introduction}

Taken together, rare diseases affect more than 350M individuals worldwide, and many are chronic, progressive, degenerative, and life-threatening. They also tend to be unfamiliar and challenging to recognize, requiring an average of five years and seven physicians to be assigned an accurate diagnosis (if diagnosed at all) [1-4]. Difficulty finding patients who are suffering from a given rare disease is a major impediment to effective diagnosis and treatment, and is an obstacle to achieving many other clinical and research goals (e.g. cohort recruitment, disease characterization, resource allocation). Because the prevalence of each rare disease is low, practically-useful patient-finding must be pursued using population-scale databases such as electronic health records (EHRs) or Web activity logs, and therefore must be realized via computational modeling [5-9]. While it is possible to derive the models through consultation with experts, this strategy can be problematic for several reasons, including the incomplete nature of experts' understanding, the complexity of disease processes, and cognitive biases associated with human decision-making [10-12,2].

These considerations motivate an interest in learning computational models for patient discovery directly from data [e.g. 13], and in particular by applying predictive modeling to EHRs [7-9,14-16]. This scheme faces difficulties of its own, perhaps the most crucial being that, with rare diseases, there are only a limited number of already-diagnosed patients available for model training. EHR databases possess additional properties which must be addressed for successful data-driven modeling, including: i.) their large-scale and high-dimensionality, ii.) the unreliable, incomplete, and sparse character of the data, iii.) the fact that patient records combine a myriad of structured (e.g. hierarchically- 
organized diagnostic codes) and unstructured (e.g. free-text notes) information, the vast majority of which is irrelevant to the prediction task at hand. See [7-9,14-16] for further discussion of these issues.

Given a target disease of interest, perhaps the most natural way to build patient-finding models is through supervised learning. Standard supervised learning in this context entails: i.) labeling the EHRs of already-diagnosed patients as being positive for the disease, ii.) adding these records to those of some unaffected patients, who form the negative class, to assemble a training dataset, and iii.) applying a validated machine learning algorithm to this training data and in this way acquire a model capable of predicting the disease status of new unseen patients [17]. Unfortunately, if the target disease is rare, then there are invariably too few patients with confirmed diagnoses to support construction of generalizable models using standard supervised techniques $[17,8]$.

Perhaps surprisingly, recent work has shown it is sometimes possible to learn accurate predictive models by combining limited labeled data with additional, less directly-informative, sources of supervision, and exploiting this 'light' supervision to guide the modeling process [18]. For instance, training examples with unreliable or missing labels can represent valuable sources of light supervision if they are appropriately leveraged [8,18-21]. Observe that this lightly-supervised approach to learning appears to represent a promising alternative in the present setting because EHR databases typically contain [8]: i.) an abundant supply of patient records which are unlabeled relative to the target disease, and ii.) patients whose records document some evidence of positive target-disease status but who do not have confirmed diagnoses, and who therefore may be assigned tentative, noisy labels.

We have developed a novel learning methodology which enables accurate patient-finding models to be induced from the imperfect real-world data encountered when investigating rare diseases. The approach enjoys two key attributes. First, learning requires only the light supervision provided by a few reliably-labeled patients, some patients with unreliable/noisy labels, and a cohort of patients who are unlabeled. Second, learning is provably safe: adding unlabeled and noisily-labeled data to the learning procedure produces models which are usually more accurate, and never less accurate, than models obtained with the reliably-labeled data alone. The safety guarantee is important. While supervision from noisy/incomplete data is intended to improve learning performance when reliably-labeled examples are limited, existing algorithms may not actually achieve this aim. Indeed, incorporating less directly-informative data often yields models that perform worse than those found using the (limited) reliable data alone [18-21].

In what follows, we describe and validate this new approach to rare-disease patient-finding. More specifically, this paper:

- introduces a new algorithm for learning prediction models from the light supervision delivered by a few reliably-labeled patients, some patients with noisy labels, and a set of patients who are unlabeled relative to the (rare) disease of interest;

- proves the algorithm is safe: the addition of unlabeled/noisily-labeled examples to a small training set of reliably-labeled data, to compensate for its limited size, yields models which are usually more accurate, and never less accurate, than corresponding models induced from the original training set;

- demonstrates that the proposed strategy outperforms state-of-the-art models for the task of detecting two different rare diseases in a country-scale database of 3.2M EHRs;

- shows the feasibility of transforming learned high-performance patient-finding models - which consist of large ensembles of complex prediction models - into simpler, but still accurate, models that are interpretable by nonexperts. 
medRxiv preprint doi: https://doi.org/10.1101/2020.07.06.20147322; this version posted July 7, 2020. The copyright holder for this preprint (which was not certified by peer review) is the author/funder, who has granted medRxiv a license to display the preprint in perpetuity. It is made available under a CC-BY-ND 4.0 International license .

\section{Safe Lightly-Supervised Learning}

\section{A. Problem Formulation}

Consider a scenario in which we are given a target rare disease (TD) and access to a database of de-identified EHRs, and wish to learn a prediction model capable of reliably detecting (undiagnosed) patients with the disease in the database. Ideally, learning should be feasible even if only a few patients known to have the disease are available for model training. To focus on the most widely-applicable setting, it is assumed the EHRs document patient encounters with general practitioners (GPs) (rather than, say, specialists) and contain standard information: basic demographic data, diagnosis codes, medication codes, free-text clinical notes, and limited lab test results (because lab test results are frequently not reported in GP EHRs). We refer to such a database as GP-EHR-DB and represent each patient in it with a vector of measurement values (e.g. age, blood glucose level) and counts of the various 'tokens' (e.g. diagnostic codes, words); see below for additional details.

As indicated above, data-driven patient-finding through supervised learning consists of three basic steps [17]:

1. model each patient as $(\mathbf{x}, y)$, where feature vector $\mathbf{x}=\left[\mathrm{x}_{1}, \ldots, \mathrm{x}_{\mathrm{d}}\right]^{\mathrm{T}} \in \mathrm{X}$ encodes information in the patient's EHR [e.g. 14] and label $y \in\{0,1\}$ denotes whether $\mathbf{x}$ has TD;

2. assemble a set of patient EHRs $L=\left\{\left(\mathbf{x}_{1}, \mathrm{y}_{1}\right), \ldots,\left(\mathbf{x}_{\mathrm{M}}, \mathrm{y}_{\mathrm{M}}\right)\right\}$, where the disease status of each of these patients is known and thus labels $\mathrm{y}_{\mathrm{i}}$ are reliable (conventionally $\mathrm{y}_{\mathrm{i}}=1$ if $\mathbf{x}_{\mathrm{i}}$ has TD);

3. use labeled dataset $\mathrm{L}$ to learn model $\mathbf{f}_{\mathrm{L}}: \mathrm{X} \rightarrow[0,1]$ which predicts the probability $\mathrm{p}(\mathrm{y}=1 \mid \mathbf{x})$ that a given patient $\mathbf{x}$ has TD [17].

A challenge with predictive modeling of rare diseases is that the number of training examples $\mathrm{M}$ is invariably small and the 'light' supervision supplied by $\mathrm{L}$ is insufficient to support effective learning $[17,8]$. In fact, dataset L may be empty $(M=0)$. It turns out this situation also can be analyzed with the proposed methodology by specifying a 'prior' for the probability that patients in GP-EHR-DB have TD (e.g. based on prevalence estimates, perhaps stratified by patient phenotype). This extension is illustrated below with one of the target rare diseases investigated in this paper.

Because (standard) supervised learning is not well-suited to the task of finding rare-disease patients, we introduce a novel strategy which exploits limited supervision to learn high-performance models, that is, models capable of accurately predicting the TD-status of all patients in GP-EHR-DB. This lightly-supervised learning (LSL) strategy integrates three sources of supervision (dataset $\mathrm{L}$ is listed again for completeness):

- reliably-labeled patients $\mathrm{L}=\left\{\left(\mathbf{x}_{1}, \mathrm{y}_{1}\right), \ldots,\left(\mathbf{x}_{\mathrm{M}}, \mathrm{y}_{\mathrm{M}}\right)\right\}$, where labels $\mathrm{y} \in\{0,1\}$ are accurate but number of patients $\mathrm{M}$ is too small to facilitate generalizable learning;

- $\quad$ noisily-labeled patients $\mathrm{N}=\left\{\left(\mathbf{x}_{\mathrm{M}+1}, \mathrm{y}^{\prime}{ }_{\mathrm{M}+1}\right), \ldots,\left(\mathbf{x}_{\mathrm{M}+\mathrm{N}}, \mathrm{y}^{\prime}{ }_{\mathrm{M}+\mathrm{N}}\right)\right\}$, where some patients in $\mathrm{N}$ are expected to be mislabeled, that is, $\mathrm{y}^{\prime} \neq \mathrm{y}$ with (unknown) probability pnoise;

- $\quad$ unlabeled patients $\operatorname{Tar}=\left\{\mathbf{x}_{\mathrm{M}+\mathrm{N}+1}, \ldots, \mathbf{x}_{\mathrm{M}+\mathrm{N}+\mathrm{T}}\right\}$; these are the patients for whom TD-status is to be predicted (i.e. the labels for these patients are unknown).

With these definitions in place, the safe LSL problem can be stated: given a target disease TD and sets of reliablylabeled, noisily-labeled, and unlabeled patient EHRs L, N, and Tar, respectively, learn a patient-finding model that normally outperforms $\mathbf{f}_{\mathrm{L}}$, learned using $\mathrm{L}$ alone, and is guaranteed to never perform worse than $\mathbf{f}_{\mathrm{L}}$. 


\section{B. Overview of Learning Method}

The proposed scheme for learning rare-disease patient-finding models in a safe lightly-supervised manner consists of three main steps. First, labeled dataset $\mathrm{L}$ is used to induce prediction model $\mathbf{f}_{\mathrm{L}}: \mathrm{X} \rightarrow \mathrm{p}_{0}$, where $\mathrm{p}_{0}$ is the 'baseline' predicted probability patient $\mathbf{x} \in$ Tar has label $\mathbf{y}=1$ (i.e., has TD). Applying $\mathbf{f}_{\mathrm{L}}$ to unlabeled dataset Tar yields a vector of predictions $\mathbf{p}_{0}=\left[\mathrm{p}_{0(\mathrm{M}+\mathrm{N}+1)}, \ldots, \mathrm{p}_{0(\mathrm{M}+\mathrm{N}+\mathrm{T})}\right]^{\mathrm{T}}$, one for each of the $\mathrm{T}$ patients in Tar.

However, if the number of labeled examples $\mathrm{M}$ is small, the predictions $\mathbf{p}_{0}$ may not be accurate. This observation motivates the second step in the learning strategy: construct an ensemble of additional predictions which can be exploited to improve upon baseline $\mathbf{p}_{0}$. Because it is advantageous for the ensemble to be diverse, we generate the predictions which comprise it using two different techniques:

- $\quad$ prediction refinement of $\mathbf{p}_{0}$ guided by the underlying structure of the data, as revealed through unsupervised clustering of unlabeled patient dataset Tar;

- noise-robust learning informed by both unreliably-labeled dataset $\mathrm{N}$ and the underlying structure of patient data.

While predictions made with these two methods are typically informative, attempting to use them to improve predictions $\mathbf{p}_{0}$ may actually decrease accuracy, rather than increase it. Thus the third step in the learning methodology is to safely combine $\mathbf{p}_{0}$ with the ensemble predictions, that is, to integrate this information to form final predictions which are ordinarily better, and never worse, than $\mathbf{p}_{0}$.

The first step in the prediction process - inducing model $\mathbf{f}_{\mathrm{L}}$ from labeled dataset $\mathrm{L}$ - is straightforward to complete using any algorithm which allows statistically-efficient supervised learning. Here we adopt a random forests (RF) model for $\mathbf{f}_{\mathrm{L}}$, with 1000 trees and default hyperparameter values [17] (varying tree number between 200 and 2000 had modest effect).

The second and third steps in the SLSL procedure are more involved, warranting the separate descriptions provided in the next two sections.

\section{Ensembles of Predictions}

This section recalls two analytic methods, which we first introduced in [22] in a different context, for building ensembles of predictions.

Prediction refinement. As indicated above, labeled dataset $L$ can be used to induce prediction model $\mathbf{f}_{\mathrm{L}}: \mathrm{X} \rightarrow \mathrm{p}_{0}$, where $\mathrm{p}_{0}$ is the predicted probability that patient $\mathbf{x} \in$ Tar has label $\mathbf{y}=1$. Applying $\mathbf{f}_{\mathrm{L}}$ to unlabeled dataset Tar yields vector of baseline predictions $\mathbf{p}_{0}=\left[\mathrm{p}_{0(\mathrm{M}+\mathrm{N}+1)}, \ldots, \mathrm{p}_{0(\mathrm{M}+\mathrm{N}+\mathrm{T})}\right]^{\mathrm{T}}$, one for each of the $\mathrm{T}$ patients in Tar. $\mathbf{p}_{0}$ can be used to create an ensemble of diverse predictions by leveraging unlabeled patients in Tar to create refinements of this baseline [22]. The assumption behind the refinement strategy is that if patients $\mathbf{x}_{\mathbf{k}}$ and $\mathbf{x}_{1}$ are 'similar' then they should possess similar labels.

More precisely, patient similarity is used to refine $\mathbf{p}_{0}$ to $\mathbf{p}^{\prime}=\left[\mathrm{p}^{\prime}{ }_{\mathrm{M}+\mathrm{N}+1}, \ldots, \mathrm{p}_{\mathrm{M}+\mathrm{N}+\mathrm{T}}\right]^{\mathrm{T}}$ with $\mathrm{p}^{\prime} \mathrm{i}$ the predicted probability instance $\mathbf{x}_{\mathrm{i}}$ has label $\mathrm{y}_{\mathrm{i}}=1$. Toward this end, let $\mathrm{La}=\mathrm{I}-\mathrm{C}$ denote a Laplacian matrix, where $\mathrm{I}$ is the identity matrix and $\mathrm{C}$ a similarity matrix computed via ensemble clustering on $\operatorname{Tar}$ [17]. $\mathrm{C}$ is constructed so that element $\mathrm{C}_{\mathrm{kl}}$ is equal to the number of times patients $\mathbf{x}_{\mathrm{k}}$ and $\mathbf{x}_{l}$ are assigned to the same cluster by members of the ensemble. (Varying the number of clusters per ensemble member and number of ensemble members has little impact on performance. Reasonable choices for these hyperparameters are 2-20 clusters and 5-20 ensemble members.) Predictions $\mathbf{p}^{\prime}$ are formed 
by balancing the goals of achieving within-cluster label similarity and maintaining agreement with the baseline predictions $\mathbf{p}_{0}$ :

$$
\min _{\mathbf{p}^{\prime}}\left\{\lambda\left\|\mathbf{p}^{\prime}-\mathbf{p}_{0}\right\|^{2}+(1-\lambda) \mathbf{p}^{\prime \mathrm{T}} \text { La } \mathbf{p}^{\prime}\right\}
$$

subject to the constraint that each $p_{i} \in[0,1]$, where $\lambda \in[0,1]$ reflects the relative expected predictive value of labeled and unlabeled data.

The optimization (1) can be accomplished by iterating the following formula over $\mathrm{j}$ until convergence (which is assured $[23,24])$ :

$$
\mathbf{p}^{\prime{ }^{j+1}}=\lambda \mathbf{p}_{0}+(1-\lambda) C_{\text {norm }} \mathbf{p}^{\prime}{ }^{\mathrm{j}}, \quad \mathbf{p}^{\prime 0}=\mathbf{p}_{0} .
$$

In (2), $\mathrm{C}_{\text {norm }}$ is the normalized version of $\mathrm{C}$ obtain by expressing $\mathrm{C}$ as a symmetric probability matrix. This solution is computationally efficient, allowing population-scale problems to be investigated.

Finally, an ensemble of R1 prediction refinements $\left\{\mathbf{p}^{\prime 1}, \ldots, \mathbf{p}^{\prime \mathrm{R} 1}\right\}$ can be assembled by varying $\lambda$ and/or defining different Laplacian matrices La through the use of distinct clusterings of Tar (see the experiments below).

Noise-robust learning. Noisily-labeled dataset $\mathrm{N}$ can also be exploited to model the probability that patient $\mathbf{x} \in$ Tar has label $y=1$. However, effectively using noisy labels requires that learning be robust to mislabeling. We achieve this robustness using a scheme similar to the one summarized in the preceding subsection (see also [8]). More precisely, 'preliminary' predictions are made for patients in Tar using a model learned from dataset $\mathrm{N}$, and these predictions are then improved using information which is independent of the noisy labels.

RFs are known to be robust to label noise [17,25], especially if built from reasonably shallow trees (larger leaf nodes can 'average out' label noise). We therefore learn 'robustified' RFs by modifying the algorithm to penalize excessive tree-depth [25]. In this way, noisily-labeled dataset $\mathrm{N}$ is used to induce prediction model $\mathbf{f}_{\mathrm{N}}: \mathrm{X} \rightarrow \mathrm{p}_{0}{ }^{\prime}$, where $\mathrm{p}_{0}{ }^{\prime}$ is the predicted probability that patient $\mathbf{x} \in$ Tar has label $\mathrm{y}=1$. Applying $\mathbf{f}_{\mathrm{N}}$ to unlabeled dataset Tar gives preliminary predictions $\mathbf{p}_{0}{ }^{\prime}=\left[\mathrm{p}_{0}{ }^{\prime}(\mathrm{M}+\mathrm{N}+1), \ldots, \mathrm{p}_{0}{ }^{\prime}(\mathrm{M}+\mathrm{N}+\mathrm{T})\right]^{\mathrm{T}}$ for the patients in Tar.

While preliminary predictions $\mathbf{p}_{0}{ }^{\prime}$ are expected to be useful, it can be helpful to mitigate the influence of label noise on $\mathbf{p}_{0}{ }^{\prime}$ with the procedure (2), rewritten here for convenience of reference (see also [25]):

$$
\mathbf{p}^{\prime \prime}{ }^{j+1}=\lambda \mathbf{p}_{0}{ }^{\prime}+(1-\lambda) C_{\text {norm }} \mathbf{p}^{\prime \prime}{ }^{j}, \quad \mathbf{p}^{\prime \prime}{ }^{0}=\mathbf{p}_{0}{ }^{\prime} .
$$

This computation yields $\mathbf{p}^{\prime \prime}=\left[\mathrm{p}^{\prime \prime}{ }_{\mathrm{M}+\mathrm{N}+1}, \ldots, \mathrm{p}^{\prime \prime}{ }_{\mathrm{M}+\mathrm{N}+\mathrm{T}}\right]^{\mathrm{T}}$, where $\mathrm{p}^{\prime \prime}{ }_{\mathrm{i}}$ is the predicted probability $\mathbf{x}_{\mathrm{i}} \in$ Tar has label $\mathrm{y}_{\mathrm{i}}=1$. As before, an ensemble of predictions $\left\{\mathbf{p}^{\prime \prime 1}, \ldots, \mathbf{p}^{\prime \prime \text { R2 }}\right\}$ can be acquired by varying $\lambda$ and/or producing a set of Laplacian matrices using distinct clusterings of Tar (see the experiments below).

\section{Algorithm SLSL}

We now show how the ensemble of predictions $\Pi=\left\{\mathbf{p}^{\prime 1}, \ldots, \mathbf{p}^{\prime \mathrm{R} 1}, \mathbf{p}^{\prime \prime 1}, \ldots, \mathbf{p}^{\prime \prime \mathrm{R} 2}\right\}$ can be leveraged to improve baseline predictions $\mathbf{p}_{0}$ made with model $\mathbf{f}_{\mathrm{L}}$ learned from (small) labeled dataset $\mathrm{L}$. Importantly, the proposed methodology is safe: the final predictions $\mathbf{p}$ are guaranteed to be at least as accurate as $\mathbf{p}_{0}$.

It is natural to approach safe LSL (SLSL), in which assurances are sought for prediction quality in the presence of uncertainty, as an adversarial learning problem [26]. Toward this end, let $\mathbf{y}=\left[\mathrm{y}_{\mathrm{M}+\mathrm{N}+1}, \ldots, \mathrm{y}_{\mathrm{M}+\mathrm{N}+\mathrm{T}}\right]^{\mathrm{T}}$ denote the true (hidden) labels for patients Tar $=\left\{\mathbf{x}_{\mathrm{M}+\mathrm{N}+1}, \ldots, \mathbf{x}_{\mathrm{M}+\mathrm{N}+\mathrm{T}}\right\}$, and define $\boldsymbol{\alpha}=\left[\alpha_{1}, \ldots, \alpha_{\mathrm{R} 1+\mathrm{R} 2}\right]^{\mathrm{T}}$ with $\alpha_{\mathrm{i}} \geq 0$ and $\Sigma_{\mathrm{i}} \alpha_{\mathrm{i}}=1 . \boldsymbol{\alpha}$ can be employed to specify various convex combinations of the predictions $\Pi$ via $\mathbf{p}_{\mathrm{c}}=\Pi \boldsymbol{\alpha}$, and in this way model an 
'adversary' within the learning process. Informally, suppose the true labels $\mathbf{y}$ satisfy $\mathbf{y}=\prod \boldsymbol{\alpha}_{\text {true }}$ for some unknown $\boldsymbol{\alpha}_{\text {true }}$. Then our objective is to form predictions $\mathbf{p}$ which are close to $\Pi \boldsymbol{\alpha}_{\text {true }}$ despite our uncertainty about $\boldsymbol{\alpha}_{\text {true. }}$. If an adversary is introduced whose goal is to choose $\alpha$ to make prediction difficult, this provides a framework within which to seek predictions $\mathbf{p}$ that are 'good' even in worst-case, that is, when $\boldsymbol{\alpha}_{\text {true }}$ is close to the adversarially-chosen value for $\alpha$.

These considerations can be formalized by stating a game theory-inspired algorithm [25-27,21] for computing predictions $\mathbf{p}$ which improve upon $\mathbf{p}_{0}$ even in worst-case.

\section{Algorithm SLSL}

1. Learn model $\mathbf{f}_{\mathrm{L}}$ from dataset $\mathrm{L}$ and use it to form baseline predictions $\mathbf{p}_{0}=\left[\mathrm{p}_{0(\mathrm{M}+\mathrm{N}+1)}, \ldots, \mathrm{p}_{0(\mathrm{M}+\mathrm{N}+\mathrm{T})}\right]^{\mathrm{T}}$ for unlabeled patient dataset $\operatorname{Tar}=\left\{\mathbf{x}_{\mathrm{M}+\mathrm{N}+1}, \ldots, \mathbf{x}_{\mathrm{M}+\mathrm{N}+\mathrm{T}}\right\}$.

2. Learn model $\mathbf{f}_{\mathrm{N}}$ from dataset $\mathrm{N}$ and use it to form preliminary predictions $\mathbf{p}_{0}{ }^{\prime}=\left[\mathrm{p}_{0}{ }^{\prime}(\mathrm{M}+\mathrm{N}+1), \ldots, \mathrm{p}_{0}{ }^{\prime}(\mathrm{M}+\mathrm{N}+\mathrm{T})\right]^{\mathrm{T}}$ for unlabeled patient dataset Tar.

3. Assemble an ensemble of predictions $\Pi=\left[\mathbf{p}^{\prime 1}, \ldots, \mathbf{p}^{\prime \mathrm{R} 1}, \mathbf{p}^{\prime \prime 1}, \ldots, \mathbf{p}^{\prime \prime \mathrm{R} 2}\right]$ by: i.) refining $\mathbf{p}_{0} \rightarrow\left\{\mathbf{p}^{\prime 1}, \ldots, \mathbf{p}^{\prime \mathrm{R} 1}\right\}$ via (2) and ii.) adapting $\mathbf{p}^{\prime} \rightarrow\left\{\mathbf{p}^{\prime \prime}, \ldots, \mathbf{p}^{\prime \prime \text { R2 }}\right\}$ using (3) (see Section 2.C for details).

4. Compute final predictions $\mathbf{p}$ by solving the following optimization problem [25-27,21]

$$
\max _{\mathbf{p}} \min _{\boldsymbol{\alpha}}\left[\left\|\mathbf{p}_{0}-\Pi \boldsymbol{\alpha}\right\|^{2}-\|\mathbf{p}-\Pi \boldsymbol{\alpha}\|^{2}\right] .
$$

It is seen that (4) quantifies the adversarial setting summarized above: we select predictions $\mathbf{p}$ to maximally improve upon $\mathbf{p}_{0}$ in worst-case, that is, when true labels $\mathbf{y}=\Pi \boldsymbol{\alpha}^{\prime}$ with $\boldsymbol{\alpha}^{\prime}$ chosen by the adversary to minimize any such improvement in prediction accuracy.

The safety and effectiveness of Algorithm SLSL is established in

Theorem SLSL: Assume there exists (unknown) $\boldsymbol{\alpha}_{\text {true }}$ such that the true labels $\mathbf{y}=\Pi \boldsymbol{\alpha}_{\text {true }}$. Then the optimal solution ( $\left.\mathbf{p}^{*}, \boldsymbol{\alpha}^{*}\right)$ to (4) ensures i.) $\left\|\mathbf{p}^{*}-\mathbf{y}\right\| \leq\left\|\mathbf{p}_{0}-\mathbf{y}\right\|$ and ii.) $\mathbf{p}^{*}$ achieves maximum worst-case performance gain over $\mathbf{p}_{0}$.

Proof: It is easy to check that objective function

$$
\Phi(\mathbf{p}, \boldsymbol{\alpha})=\left\|\mathbf{p}_{0}-\Pi \boldsymbol{\alpha}\right\|^{2}-\|\mathbf{p}-\Pi \boldsymbol{\alpha}\|^{2}
$$

satisfies the conditions of the minimax theorem [27], so that

$$
\max _{\mathbf{p}} \min _{\alpha} \Phi(\mathbf{p}, \boldsymbol{\alpha})=\min _{\alpha} \max _{\mathbf{p}} \Phi(\mathbf{p}, \boldsymbol{\alpha}) .
$$

This, in turn, implies

$$
\Phi\left(\mathbf{p}, \boldsymbol{\alpha}^{*}\right) \leq \Phi\left(\mathbf{p}^{*}, \boldsymbol{\alpha}^{*}\right) \leq \Phi\left(\mathbf{p}^{*}, \boldsymbol{\alpha}\right) .
$$

Setting $\mathbf{p}=\mathbf{p}_{0}$ and $\boldsymbol{\alpha}=\boldsymbol{\alpha}_{\text {true }}$ in this chain of inequalities gives

$$
\begin{aligned}
\Phi\left(\mathbf{p}_{0}, \boldsymbol{\alpha}^{*}\right) & =\left\|\mathbf{p}_{0}-\Pi \boldsymbol{\alpha}^{*}\right\|^{2}-\left\|\mathbf{p}_{0}-\Pi \boldsymbol{\alpha}^{*}\right\|^{2} \\
& =0 \leq\left\|\mathbf{p}_{0}-\Pi \boldsymbol{\alpha}_{\text {true }}\right\|^{2}-\left\|\mathbf{p}^{*}-\Pi \boldsymbol{\alpha}_{\text {true }}\right\|^{2},
\end{aligned}
$$

implying $\left\|\mathbf{p}^{*}-\mathbf{y}\right\| \leq\left\|\mathbf{p}_{0}-\mathbf{y}\right\|$, which is claim i. Claim ii. follows from this and the optimality of $\mathbf{p}^{*}$. 
Thus predictions $\mathbf{p}^{*}=\left[\mathrm{p}^{*}{ }_{\mathrm{M}+\mathrm{N}+1}, \ldots, \mathrm{p}^{*}{ }_{\mathrm{M}+\mathrm{N}+\mathrm{T}}\right]^{\mathrm{T}}$ for the TD-status of patients Tar $=\left\{\mathbf{x}_{\mathrm{M}+\mathrm{N}+1}, \ldots, \mathbf{x}_{\mathrm{M}+\mathrm{N}+\mathrm{T}}\right\}$ are always at least as close to the true labels $\mathbf{y}$ as baseline predictions $\mathbf{p}_{0}$, and improve upon $\mathbf{p}_{0}$ to the maximum extent possible in worst-case (when $\mathbf{y}=\Pi \boldsymbol{\alpha}^{*}$ ).

In addition to being provably-safe, Algorithm SLSL is efficient to execute. For instance, it is easy to show that optimization problem (4) is convex, implying that optimal predictions $\mathbf{p}^{*}$ can be computed via standard convex quadratic programming [28].

\section{Patient-Finding Experiments}

We now describe a study in which Algorithm SLSL is deployed to find patients suffering from two very rare, difficult-to-diagnose TDs in a large GP-EHR-DB. The investigation:

- $\quad$ performs patient-finding for two quite different TDs in settings in which reliable labels are extremely limited (2 and 0 patients with confirmed diagnoses for the two TDs);

- illustrates construction of EHR training datasets in this challenging scenario;

- demonstrates that models learned with Algorithm SLSL outperform state-of-the-art benchmark models and enable high-precision detection of TD patients in a country-scale database of 3.2M EHRs;

- identifies those patient features which possess significant predictive power for each TD;

- $\quad$ illustrates the way results of chart review by TD specialists can be leveraged to obtain improved training labels and permit induction of even more accurate patient-finding models.

\section{A. Setup}

The main goal of these experiments is to learn models which can accurately detect patients affected by a rare disease of interest using standard GP EHR data. In order to assess the generality of the approach, the experiments consider two TDs with quite distinct attributes:

- $\quad$ acute hepatic porphyria (AHP), an autosomal dominant disorder impacting heme synthesis and associated with serious and potentially life-threatening neurovisceral attacks; clinical presentation is heterogeneous and can include severe abdominal pain, nausea, vomiting, hypertension, tachycardia, and various neurological and psychiatric symptoms; prevalence is estimated to be around 5-20/1M [29,30];

- lipodystrophy (LD), a genetic/acquired disorder characterized by selective deficiencies of adipose tissue and associated with serious complications including diabetes mellitus, dyslipidemia, liver disease, heart disease, pancreatitis, and reproductive dysfunction; prevalence is estimated to be approximately 2-4/1M [31,32].

GPs may be unfamiliar with these diseases, and clinical manifestation of each is variable and can mimic many other more common diseases. Thus these TDs are often repeatedly misdiagnosed or not detected at all. Difficulty identifying patients leads to treatment delays, negatively impacts patient health, and slows progress in clinical research [14], highlighting the real-world relevance of this set of experiments.

Experiments were conducted using a database of 3.2M de-identified EHRs arising from patient encounters with GPs in a European country [33]; we refer to this database as GP-EHR-DB-NL. The information contained in GP-EHRDB-NL includes basic demographics data, ICPC diagnosis and symptom codes (http://www.who.int/classifications/ 
icd/adaptations/icpc2/), ATC drug codes (https://www.whocc.no/atc ddd index/), and limited free text clinical notes and lab results. GP-EHR-DB-NL is representative of other de-identified GP EHR datasets and the challenges faced when analyzing them. For instance, clinical notes and lab test records are typically brief and incomplete, reflecting privacy concerns and the nature of GP visits.

Patients are represented as pairs $(\mathbf{x}, \mathbf{y})$, where feature vector $\mathbf{x}$ encodes a patient's EHR information in the usual way. Briefly, $\mathbf{x}$ is composed of year-of-birth, gender, counts for all ICPC and ATC codes, numerical values for lab test results and clinical measurements, and a 'bag-of-words' model for free-text clinical notes (yielding high-dimensional patient vectors). Further details on this processing step can be found in [e.g. 14,8]. The label y designates TD-status, with $\mathrm{y}=1$ if the patient has the TD in question and $\mathrm{y}=0$ if not.

Training datasets were assembled in the following manner. For each TD, the positive-class examples consist of patients with confirmed diagnoses (2 for AHP, 0 for LD) together with a number of (noisily-labeled) patients who may have TD (i.e., their EHRs contain some evidence of TD). The negative class training set was gathered by randomlysampling EHRs of i.) patients diagnosed with diseases having clinical presentation similar to TD (mimics), ii.) patients matched to positive-class examples for age, gender, and date of earliest record. The criteria employed for identifying positive-class patients were defined through consultation with domain experts [8]. To avoid data leakage, only information entered into an EHR prior to diagnosis of the pertinent condition (TD or mimic disorder) was retained for analysis. Similarly, all patient EHRs were edited to remove mentions of terms used in the dataset collection procedure.

Attributes of the training datasets are listed in Table 1. Notice that each TD has low prevalence, there are few/no already-diagnosed patients in GP-EHR-DB-NL, and patients are described by thousands of features. As mentioned, the noise and high-dimensionality of the dataset are key obstacles to learning accurate, generalizable models for rare disease patient-finding.

Table 1. Training datasets.

\begin{tabular}{|c|c|c|c|c|c|c|}
\hline $\begin{array}{c}\text { Target } \\
\text { Disease } \\
\text { (TD) }\end{array}$ & $\begin{array}{c}\text { Estimated } \\
\text { Prevalence }\end{array}$ & $\begin{array}{c}\text { \# Confirmed } \\
\text { Diagnosed } \\
\text { Patients }\end{array}$ & $\begin{array}{c}\text { \# Positive } \\
\text { Class Patients: } \\
\text { TD-Similar }\end{array}$ & $\begin{array}{c}\text { \# Negative } \\
\text { Class Patients: } \\
\text { Mimics }\end{array}$ & $\begin{array}{c}\text { \# Negative } \\
\text { Class Patients: } \\
\text { Matched }\end{array}$ & \# Features \\
\hline AHP & $5-20 / 1 M$ & 2 & 50 & 100 & 100 & 7469 \\
\hline LD & $2-4 / 1 M$ & 0 & 12 & 100 & 100 & 7469 \\
\hline
\end{tabular}

In both the AHP and LD patient-finding experiments, Algorithm SLSL is implemented in four steps as specified in Section 2.D and detailed below.

1. Baseline prediction $\mathbf{p}_{0}$ is made with an RF model $\mathbf{f}_{\mathrm{L}}: \mathrm{X} \rightarrow \mathrm{p}_{0}$ learned from dataset L (1000 trees and default hyperparameters; see Section 2.B and [17]). 
medRxiv preprint doi: https://doi.org/10.1101/2020.07.06.20147322; this version posted July 7, 2020. The copyright holder for this preprint (which was not certified by peer review) is the author/funder, who has granted medRxiv a license to display the preprint in perpetuity. It is made available under a CC-BY-ND 4.0 International license .

2. Preliminary prediction $\mathbf{p}_{0}{ }^{\prime}$ is formed with a noise-robust $\mathrm{RF} \mathbf{f}_{\mathrm{N}}: \mathrm{X} \rightarrow \mathrm{p}_{0}{ }^{\prime}$ learned from dataset $\mathrm{N}$ (see Section 2.C and [8]).

3. Ensemble predictions $\Pi=\left[\mathbf{p}^{\prime 1}, \mathbf{p}^{\prime 2}, \mathbf{p}^{\prime 3}, \mathbf{p}^{\prime \prime 1}, \mathbf{p}^{\prime \prime 2}, \mathbf{p}^{\prime \prime 3}\right]$ are made by: i.) refining $\mathbf{p}_{0} \rightarrow\left\{\mathbf{p}^{\prime 1}, \mathbf{p}^{\prime 2}, \mathbf{p}^{\prime 3}\right\}$ via (2) with three clusterings of Tar (k-means method [17] with $\mathrm{k} \in\{2,4,7\}$ ) and ii.) adapting $\mathbf{p}_{0}{ }^{\prime} \rightarrow\left\{\mathbf{p}^{\prime \prime 1}, \mathbf{p}^{\prime \prime 2}, \mathbf{p}^{\prime \prime 3}\right\}$ using (3) with three clusterings of Tar (k-means, $\mathrm{k} \in\{2,4,7\}$ ). Hyperparameter $\lambda$ is set to $\lambda=0.6$ in iterative algorithms (2) and (3) (selected through nested cross-validation [17]).

4. Final prediction $\mathbf{p}^{*}$ is made by solving $\max _{\mathbf{p}} \min _{\alpha}\left[\left\|\mathbf{p}_{0}-\Pi \boldsymbol{\alpha}\right\|^{2}-\|\mathbf{p}-\Pi \boldsymbol{\alpha}\|^{2}\right]$ using standard convex quadratic programming [28] (see Section 2.D).

Because intermediate and final predictions generated by Algorithm SLSL can be computed efficiently [23-25,28], patient-finding can be conducted using country-scale EHR databases.

To permit an objective, quantitative assessment of the performance of Algorithm SLSL, we compare the accuracy of its predictions with those of two benchmark models:

- 'Anchor and learn algorithm' [34,35]: a state-of-the-art technique for learning disease-state estimation models with very limited labeled data;

- 'TD-training' model: assume the TD training dataset is correctly-labeled and use all positive-class patients (i.e., the combination of already-diagnosed patients and patients for whom evidence exists of positive disease-status) as predictions for which patients have TD; this 'model' is similar to standard patient-finding methodologies, in which expert-derived code/keyword queries are employed to identify candidate patients [8]; its use here also allows the noise-level of training labels to be quantified.

We considered using standard supervised learning for this task (support vector machines, L1-regularized logistic regression [17]) but these models performed poorly in a pilot examination, likely because of the limited, noisy nature of the training data.

\section{B. Results}

Algorithm SLSL is now applied to the task of finding undiagnosed TD patients in country-scale EHR database GPEHR-DB-NL. Patient-finding is implemented in three steps:

1. Replace missing EHR fields with a 'missing' token [17].

2. Train Algorithm SLSL using the training dataset assembled for the TD under study (AHP or LD, see Table 1). Use the learned model to predict the probability that each patient in GP-EHR-DB-NL has the TD.

3. Rank-order patients by predicted probability of having TD and extract the Top 20 and Top CP candidates (with the threshold CP determined based upon natural breakpoints in the probabilities).

For each TD, we recruited appropriate specialists practicing in Europe to review the EHRs of the top CP candidates returned by our models. To increase reliability and objectivity of the reviews, the order of the patients was shuffled and 10 randomly-chosen patients were added to each list [8]. The specialists classified each candidate as 'likely TD', 'possible TD', 'unlikely TD', 'highly unlikely TD', 'not TD', or 'unable to assess'. The results of the reviews are reported in Tables 2 and 3. Taken together, these results demonstrate that Algorithm SLSL is able to learn accurate patient-finding models from light-supervision provided by a few (two or zero) labeled examples together with noisi- 
ly-labeled/unlabeled data. Indeed, the Top 20 Lists for AHP and LD achieve 90\% precision and 70\% precision, respectively, substantially outperforming state-of-the-art in this setting. To place these results in context, observe from current prevalence data (Table 1 ) that $\sim 0.001 \%$ of patients are expected to have AHP and $\sim 0.0003 \%$ of patients are expected to have LD.

In more detail, rank-ordering all patients in GP-EHR-DB-NL according to predicted probability of having AHP reveals the top 42 candidates have qualitatively higher AHP-probability than the rest ( 0.9). The AHP specialists reviewing the EHRs for these patients classified 17 as 'likely AHP' and an additional 16 as 'possible AHP', for a total of 33 plausible candidates (79\% of the list). The top 20 list has even better precision, containing 14 'likely AHP' patients and 4 'possible AHP' patients, for a total of 18 plausible candidates ( $90 \%$ precision @ 20). In comparison, the Anchor and Learn model [34,35] nominates 33 potential AHP patients, of whom 12 are deemed to be plausible candidates (36\%), while the 50-patient AHP-training list includes 14 plausible candidates (28\%). The latter result shows that, as hypothesized, Algorithm SLSL can learn prediction models which are more accurate than the labels used for training. It can be seen that the models learned with Algorithm SLSL are also reasonably well-calibrated: the top 20 list has a greater ratio of 'likely AHP' to 'possible AHP' patients than the top 42 list. Se Table 2 for a summary of the experimental results for AHP.

Table 2. Results of patient-finding experiments: AHP.

\begin{tabular}{|l|c|c|c|c|c|}
\hline & $\begin{array}{c}\text { Total } \\
\text { Candidates (K) }\end{array}$ & $\begin{array}{c}\text { Likely } \\
\text { AHP }\end{array}$ & $\begin{array}{c}\text { Possible } \\
\text { AHP }\end{array}$ & $\begin{array}{c}\text { Plausible } \\
\text { Candidates }\end{array}$ & $\begin{array}{c}\text { Precision } \\
\text { @ K }\end{array}$ \\
\hline Top 20 List & 20 & 14 & 4 & 18 & $90 \%$ \\
\hline Top 42 List & 42 & 17 & 16 & 33 & $79 \%$ \\
\hline AHP-Training List & 50 & 6 & 8 & 14 & $28 \%$ \\
\hline Anchor and Learn & 33 & 6 & 6 & 12 & $36 \%$ \\
\hline
\end{tabular}

By rank-ordering patients in GP-EHR-DB-NL according to predicted probability of having LD we find that the top 36 candidates have qualitatively higher LD-probability than the rest $(\sim 0.8)$. This list of 36 candidates includes 4 patients that the specialist assessed to be 'likely LD' and 15 more deemed 'possible LD', for a total of 19 plausible candidates (53\% of the list). The top 20 list has higher precision, containing the 4 'likely LD' patients and 10 'possible LD' patients, for a total of 14 plausible candidates (70\% precision @ 20). As is the case with AHP, the Anchor and Learn method [34,35] provides much poorer performance, nominating 15 potential LD patients of whom 5 are deemed plausible candidates (33\%). The 12-patient LD-training list includes 3 plausible candidates (25\%), again indicating that Algorithm SLSL is capable of learning a prediction model which is more accurate than its training data. See Table 3 for a summary of the experimental results for LD.

Knowing which features captured in EHRs are predictive of a given rare disease is of value in various clinical applications. We assessed feature predictive power using both forward- and backward-stepwise analyses [17], and now highlight a few findings. The predictive power of structured v. unstructured features varies across the two TDs, with ICPC codes having significant predictive power for AHP, free text clinical notes being quite helpful for LD, and lab 
test results, though limited in these GP EHRs, being predictive for both AHP and LD. Proxies for temporal order of events are useful for distinguishing AHP from its mimics. Unusual combinations of otherwise common symptoms are also predictive (e.g. for LD, diabetes presenting in patients with normal or low body mass index (BMI)).

Fairly subtle characteristics of 'patient journey' can be informative. For example, longer sequences of unique ICPC codes, conjectured to reflect GP uncertainty/evolving understanding when confronted with a rare disorder, are predictive features for both AHP and LD, as are notes documenting GP behavior arising from uncertainty/unfamiliarity (e.g. multiple referrals or consultations). Finally, mental health-related clinical notes and medications (e.g. terms related to depression and anxiety, ATC codes/notes for anti-anxiety medications and anti-depressants) are distinguishing for both AHP and LD.

Table 3. Results of patient-finding experiments: LD.

\begin{tabular}{|l|c|c|c|c|c|}
\hline & $\begin{array}{c}\text { Total } \\
\text { Candidates (K) }\end{array}$ & $\begin{array}{c}\text { Likely } \\
\text { LD }\end{array}$ & $\begin{array}{c}\text { Possible } \\
\text { LD }\end{array}$ & $\begin{array}{c}\text { Plausible } \\
\text { Candidates }\end{array}$ & $\begin{array}{c}\text { Precision } \\
\text { @ K }\end{array}$ \\
\hline Top 20 List & 20 & 4 & 10 & 14 & $70 \%$ \\
\hline Top 36 List & 36 & 4 & 15 & 19 & $53 \%$ \\
\hline LD-Training List & 12 & 0 & 3 & 3 & $25 \%$ \\
\hline Anchor and Learn & 15 & 1 & 4 & 5 & $33 \%$ \\
\hline
\end{tabular}

\section{Model Retraining with Specialist Labels}

The experiments summarized in the preceding section demonstrate the accuracy and utility of patient-finding models learned by applying Algorithm SLSL to noisily-labeled/unlabeled training data. Interestingly, the chart review conducted by TD specialists in support of algorithm evaluation affords the opportunity to improve upon these models by leveraging TD-specialist appraisals. More specifically, we can replace the noisily-labeled positive-class training examples employed above with patient EHRs classified as 'plausible TD' by the specialists (i.e. 'likely TD' and 'possible TD' for each TD). Because the revised positive-class training sets are small, consisting of 33 and 19 patients for AHP and LD, respectively, Algorithm SLSL is still used for model-building (e.g. to permit unlabeled examples to be exploited).

This strategy does indeed generate very high-quality models. For instance, ten-fold cross-validation runs of the resulting models yield the following estimates for out-of-sample area under ROC curve (AUC):

- $\quad$ for $\mathrm{AHP}, \mathrm{AUC}=0.98$;

- $\quad$ for $\mathrm{LD}, \mathrm{AUC}=0.96$.

As another test of the new model, we rank-ordered the 'top 42' and 'top 36' lists for AHP and LD, respectively, according to the predicted TD-probabilities returned by the retrained patient-finding models. The reordered lists agree more closely with the specialists’ assessments. 


\section{Interpretable Models}

\section{A. Basic Idea}

The preceding theoretical (Section 2) and empirical (Section 3) discussions demonstrate that Algorithm SLSL offers a powerful and broadly-applicable approach to finding rare-disease patients in EHR databases. However, while the models learned by Algorithm SLSL are accurate, they are not interpretable by non-experts: each patient's predicted TD-status is obtained by combining outputs of thousands of individual supervised and unsupervised models through convex quadratic programming. It would be desirable to use the algorithm as the basis for deriving detection models which are both accurate and interpretable [36-38], as this would enable clinicians to understand, and therefore trust, a model's prediction process.

This section explores the feasibility of transforming the high-performance prediction models produced by Algorithm SLSL into accurate, interpretable models. The proposed technique accommodates key challenges associated with the clinical domain. Predictions concerning patient disease status are typically high-consequence and thus demand reliable, validated models. In addition, EHR data is characterized by missing, noisy, hierarchically-related features [3941]. These problem requirements and attributes represent key obstacles for standard ways of achieving model interpretability. For instance, one common idea is to achieve interpretability by trading-off model performance for simplicity. However, the resulting models generally do not meet the performance demands of clinical tasks [37,38]. Alternatively, insights can be gained into the workings of a complex model by identifying which features are important to its predictions. Unfortunately, feature importance cannot be reliably-quantified for models that are designed to handle missing/noisy data or hierarchically-organized features [38-41].

We now present a simple method for learning accurate, interpretable patient-finding models which overcomes these challenges. The approach operationalizes a reasonable, mathematically-sound definition for model interpretability: a model is deemed interpretable if a user can take the model's input data and parameters and, in reasonable time, execute the calculations necessary to make a prediction [36,37]. The basic algorithm is composed of three steps.

\section{Algorithm IM (Interpretable Modeling)}

Given a target prediction task, interpretable models are learned in three steps.

1. Assemble a training set of patient EHRs (e.g. cohorts of patients known/suspected to either have or not-have the disease of interest).

2. Learn full model $\mathrm{M}_{\mathrm{F}}$ which is capable of forming accurate predictions for new patients despite the practical difficulties associated with EHR-based modeling (e.g. missing feature values, unreliable patient labels, mix of structured and unstructured data) [8].

3. Abstract the full model $\mathrm{M}_{\mathrm{F}}[42,43]$ to produce interpretable decision tree model $\mathrm{M}_{\mathrm{IDT}}$ using one of the following methods:

a. fit a decision tree to the predictions of $\mathrm{M}_{\mathrm{F}}$ (rather than the patient labels appearing in the training dataset);

b. abstract $\mathrm{M}_{\mathrm{F}}$ using a slight extension of the logic-preserving (abstraction) algorithm put forth in [43].

A schematic of the procedure is depicted in Figure 1. In this feasibility study, only the model-abstraction 3a. is used; a more comprehensive investigation of model-abstraction and interpretable modeling, including the use of technique 3b., may be found in [38] (see also [43]). 


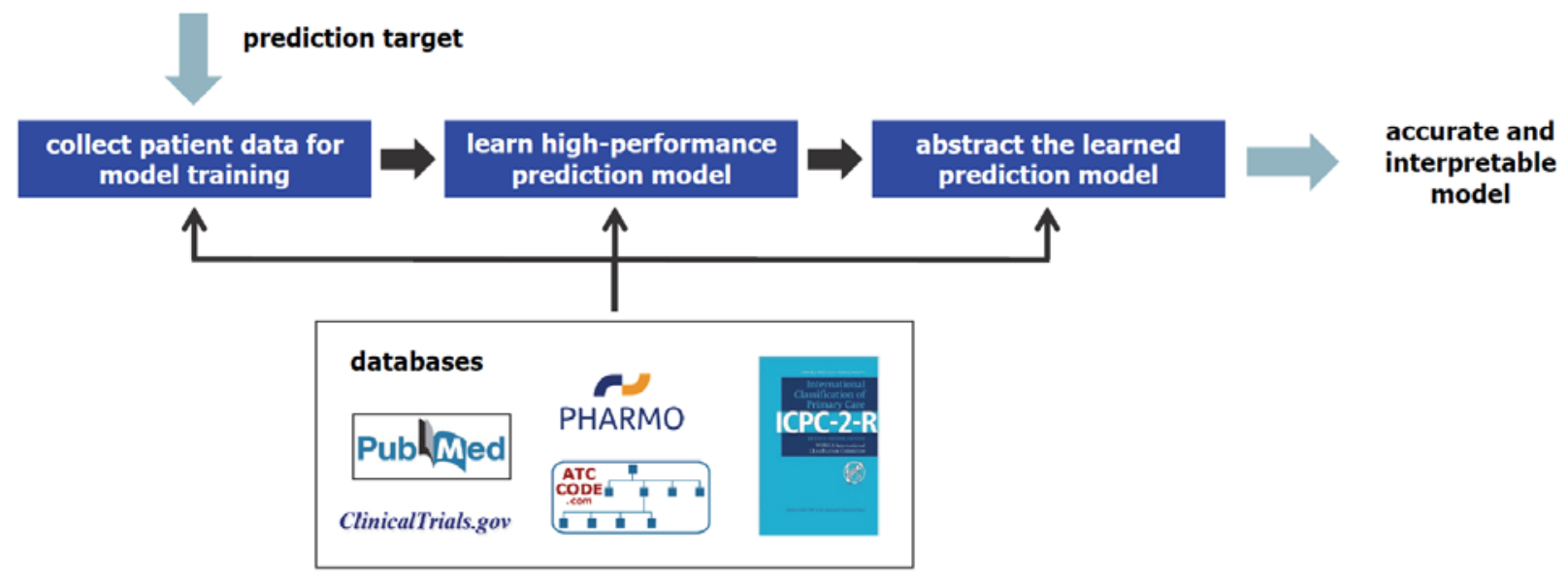

Figure 1. Schematic of interpretable modeling process.

While it is possible to apply the proposed interpretable-modeling procedure even with TDs that possess heterogeneous presentations spanning diverse medical domains, the resulting decision trees can be too complex to be readilycomprehensible. To address this issue we have designed an extension to the basic process, in which an ensemble of 'specialist' decision trees, rather than a single tree, is learned. For a given TD, the ensemble of specialists is induced by partitioning the full feature space into separate subspaces and learning one tree for each subspace [38]. The selection of feature subspaces is guided by the structure of medical domains, and consequently a learned ensemble tends to be composed of models which i.) align with medical specialties (e.g. cardiology, oncology), or ii.) correspond to aspects of the patient journey (e.g. by recognizing that GPs are imperfect 'sensors' and identifying feature subspaces that reflect clinical confusion). Interpretable specialist ensembles are illustrated in the next section and discussed in detail in [38].

\section{B. Experiments}

The main goal of the experimental study is to evaluate the possibility of learning patient-finding models which are simultaneously accurate and interpretable. The task of interest is to detect undiagnosed AHP and LD patients in GPEHR-DB-NL (3.2M patients). Algorithm IM is implemented as specified in Section 4.A. For each TD, model $\mathrm{M}_{\mathrm{F}}$ is constructed by

- learning an initial model $\mathrm{M}_{\mathrm{init}}$ using Algorithm SLSL;

- $\quad$ submitting patients predicted to have TD by $\mathrm{M}_{\text {init }}$ to disease specialists for chart review and revising the labels of the (positive-class) training dataset based upon this specialist assessment;

- $\quad$ training model $\mathrm{M}_{\mathrm{F}}$ using the revised data;

- note: recall that models $\mathrm{M}_{\mathrm{F}}$ are high-performance, achieving cross-validated accuracies of AUC $=0.98$ for AHP and AUC $=0.96$ for LD, but are too complex to allow convenient inspection of their decision-logic. 
An interpretable model, denoted $\mathrm{M}_{\mathrm{IDT}}$, is then obtained by fitting a CART decision tree to predictions of the learned full model $\mathrm{M}_{\mathrm{F}}$ (see e.g. [17] for background on CART models).

Figure 2 displays two decision tree models for LD patient-finding generated using Algorithm IM with two different choices of hyperparameters [38,17]. Each model is accurate, realizing out-of-sample AUC $>0.9$ on the training set. Moreover, the models are interpretable: their prediction-logic is straightforward to understand and they offer insight into the way LD presents in GP EHRs. For example, it is seen that LD patients are characterized by high blood glucose and triglycerides (TRI), as expected, and tend to have comorbidities (e.g. diabetes) that are unusual for individuals with normal/low BMI. Perhaps more surprising, presence of 'clinical confusion' in a patient's record, quantified using counts of unique diagnostic codes or consultations, is found to be a useful predictor of LD.
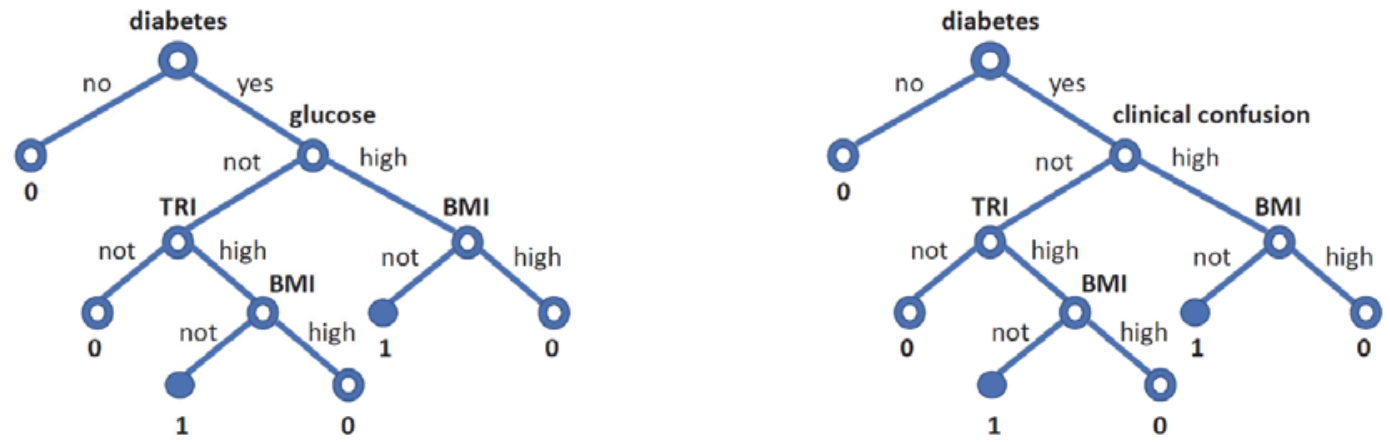

Figure 2. Sample interpretable models for LD patient-finding.

These results broadly agree with, and appear to supplement and increase the utility of, published guidelines for diagnosing LD in clinical settings. For instance, standard guidelines often begin with 'suspicion of LD' [e.g. 31], which reduces their value to GPs (e.g. there are over 7000 rare diseases, so it may be impractical to require that a GP suspect one of them in order to start the diagnostic process). Papers which may help clinicians 'suspect LD' [e.g. 44] have some decision points in common with our models $\mathrm{M}_{\mathrm{IDT}}$ (e.g. diabetes with normal BMI, high triglycerides) but also miss informative predictors (clinical confusion) and do not specify the decision-logic necessary for implementation.

Because AHP has a heterogeneous clinical presentation [29,30], we built an ensemble of specialist trees to form the interpretable model $\mathrm{M}_{\mathrm{IDT}}$ for this disease. As an illustrative example, Figure 3 depicts a three-tree ensemble induced by partitioning the full feature space into different subspaces and learning ensemble members on the individual subspaces [38]. It can be seen the individual models either align with medical specialties, such as psychiatry, or reflect features determined to be predictive via the learning process (e.g. feature subspaces associated with a GP examination).

In particular, the tree at top-left of Figure 3 forms predictions by combining well-known diagnostic and demographic information (e.g. abdominal pain, peripheral neuropathy, year of birth) with proxies for clinical confusion (such lengthy clinical notes, numerous disparate medication prescriptions), and thereby encodes 'GP as an imperfect sensor' data. The other trees serve to bring 'labs data', 'psychiatric interactions', and suspicion of porphyria (captured 
medRxiv preprint doi: https://doi.org/10.1101/2020.07.06.20147322; this version posted July 7, 2020. The copyright holder for this preprint (which was not certified by peer review) is the author/funder, who has granted medRxiv a license to display the preprint in perpetuity. It is made available under a CC-BY-ND 4.0 International license .

in ICPC codes) into the patient-finding process. Taken together, the ensemble of trees is accurate, providing out-ofsample AUC $>0.9$ on the training set, yet interpretable: clinicians can understand each specialist decision trees and the way in which the trees 'vote' to make a prediction regarding AHP status.

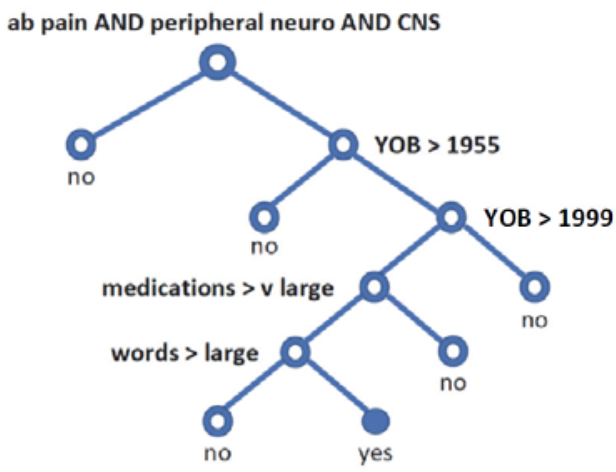

'GP-as-sensor' specialist

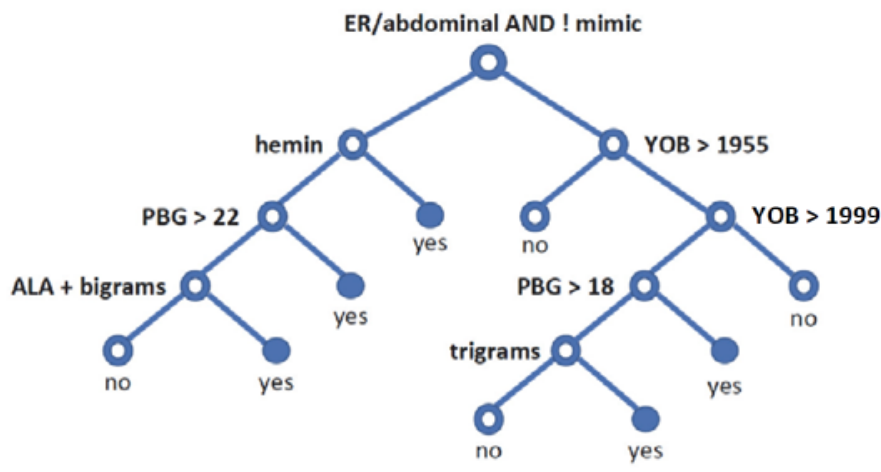

'labs' specialist

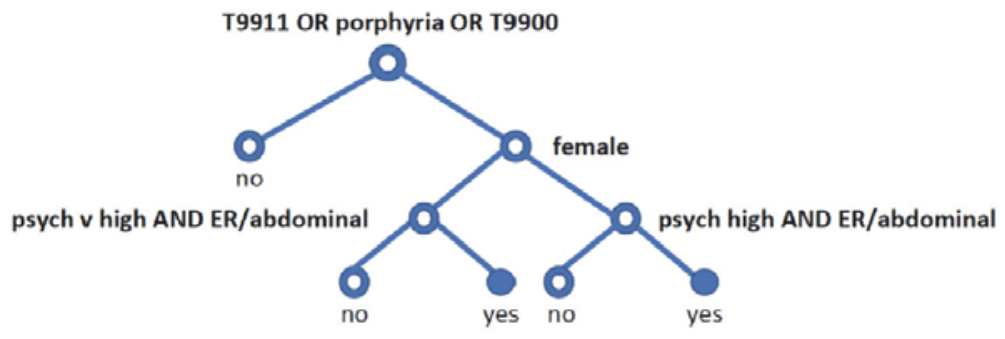

psychiatry specialist

Figure 3. Sample ensemble of interpretable models for AHP patient-finding.

\section{Concluding Remarks}

This paper presents a novel lightly-supervised approach to learning models with which to detect rare disease patients in EHR databases. The proposed algorithm leverages unlabeled and unreliably-labeled EHR data to induce accurate models from limited numbers of 'clean' labels, thereby addressing a key challenge with rare disease patient-finding. We prove the algorithm is safe: adding unlabeled/unreliably-labeled data to the learning procedure produces models which are usually more accurate, and never less accurate, than models learned from the reliably-labeled data alone. Moreover, the method is shown to substantially outperform state-of-the-art models in 'real-world' patient-finding experiments involving two different rare diseases and a database of 3.2M GP EHRs. The high-performance models generated through light supervision can be transformed into simpler models that are simultaneously accurate and easily-understandable by non-experts. 
medRxiv preprint doi: https://doi.org/10.1101/2020.07.06.20147322; this version posted July 7, 2020. The copyright holder for this preprint (which was not certified by peer review) is the author/funder, who has granted medRxiv a license to display the preprint in perpetuity. It is made available under a CC-BY-ND 4.0 International license .

\section{References}

1. Bloss, $\mathrm{S}$ et al., 'Diagnostic needs for rare diseases and shared prediagnostic phenomena', PLoS ONE, Vol. 12, 2017.

2. Zurynski, Y et al., 'Rare disease: A national survey of pediatricians' experiences and needs', BMJ Pediatrics, Vol. 1, 2017.

3. The Global Challenge of Rare Disease Diagnosis, Shire, 2018.

4. Boycott, $\mathrm{K}$ and D Ardigo, 'Addressing challenges in the diagnosis and treatment of rare genetic diseases', $\mathrm{Na-}$ ture Reviews Drug Discovery, Vol. 17, 2018.

5. Svenstrup, D et al., 'Rare disease diagnosis: A review of web search, social media, and large-scale data mining approaches’, Rare Diseases, Vol. 3, 2015.

6. Richesson, $\mathrm{R}$ et al., 'Clinical phenotyping in selected national networks: Demonstrating the need for highthroughput, portable, and computational methods', AI in Medicine, Vol. 71, 2016.

7. Shen, $\mathrm{F}$ et al., 'Leveraging collaborative filtering to accelerate rare disease diagnosis', AMIA Annual Symposium, Washington, DC USA, November 2017.

8. Colbaugh, $\mathrm{R}$ et al., 'Learning to identify rare disease patients from electronic health records', AMIA Annual Symposium, San Francisco, CA USA, November 2018.

9. Garcelon, $\mathrm{N}$ et al., 'Next generation phenotyping using narrative reports in a rare disease clinical data warehouse', J Rare Diseases, Vol. 13, 2018.

10. Elstein, A and A Schwarz, 'Clinical problem solving and diagnostic decision making: Selective review of the cognitive literature’, BMJ, Vol. 325, 2002.

11. Evans, W and I Rafi, 'Rare diseases in general practice’, British J. General Practice, Vol. 66, 2016.

12. Norman, $\mathrm{G}$ et al., 'The causes of errors in clinical reasoning: Cognitive biases, knowledge deficits, and dual process thinking', Academic Medicine, Vol. 92, 2017.

13. White, $\mathrm{R}$ and $\mathrm{E}$ Horvitz, 'Evaluation of the feasibility of screening patients for early signs of lung carcinoma in web search logs’, JAMA Oncology, Vol. 3, 2017.

14. Miotto, R et al., 'Deep Patient: An unsupervised representation to predict the future of patients from the electronic health records', Scientific Reports, Vol. 6, 2016.

15. Goldstein, B et al., 'Opportunities and challenges in developing risk prediction models with electronic health records data: A systematic review', J AMIA, Vol. 24, 2017.

16. Jia, J et al., 'RDAD: A machine learning system to support phenotype-based rare disease diagnosis', Front Genetics, Vol. 9, 2018.

17. Hastie, T, R Tibshirani, and J Friedman, The Elements of Statistical Learning, Springer, New York, 2009.

18. Zhou, Z-H, 'A brief introduction to weakly supervised learning', National Science Review, Vol. 5, 2018.

19. Li, Y-F and Z-H Zhou, 'Towards making unlabeled data never hurt', IEEE Trans PAMI, Vol. 37, 2015.

20. Balsubramani, A and Y Freund, 'Optimally combining classifiers using unlabeled data', COLT, Paris, France, July 2015.

21. Gou, L-Z and Y-F Li, 'A general formulation for safely exploiting weakly supervised data', AAAI, New Orleans, LA USA, February 2018.

22. Colbaugh, $\mathrm{R}$ et al., 'Ensemble transfer learning for Alzheimer's disease diagnosis', IEEE EMBC, Seoul, South Korea, July 2017.

23. Colbaugh, R, 'Predictability of human behavior', Invited CISAC Science Seminar, Stanford University, April 2012.

24. Beck, A and L Tetruashvili, 'On the convergence of block coordinate descent type methods', SIAM J Optimization, Vol. 23, 2013.

25. Colbaugh, $\mathrm{R}$ and $\mathrm{K}$ Glass, 'Predicting antimicrobial resistance via lightly-supervised learning', IEEE SMC, Bari, Italy, October 2019. 
medRxiv preprint doi: https://doi.org/10.1101/2020.07.06.20147322; this version posted July 7, 2020. The copyright holder for this preprint (which was not certified by peer review) is the author/funder, who has granted medRxiv a license to display the preprint in perpetuity. It is made available under a CC-BY-ND 4.0 International license.

26. Colbaugh, R and K Glass, 'Predictability-oriented defense against adaptive adversaries', IEEE SMC, Seoul, Korea, October 2012.

27. Webb, J, Game Theory, Springer, London, 2007.

28. Boyd, S and L Vandenberghe, Convex Optimization, Cambridge University Press, UK 2004.

29. Desnick, R, ‘Clinical Overview of the Acute Hepatic Porphyrias’, preprint, 2017.

30. Anderson, $\mathrm{K}$ et al., 'Recommendations for the diagnosis and treatment of the acute porphyrias', Annals of Internal Medicine, Vol. 142, March 2005.

31. Savage, D, 'Lipodystrophy: Disease Background', preprint, December 2013.

32. Brown, $\mathrm{R}$ et al., 'The diagnosis and management of lipodystrophy syndromes: A multi-society practice guideline', J Clinical Endocrinology and Metabolism, Vol. 101, December 2016.

33. PHARMO Database Network, www.pharmo.com, last accessed December 2019.

34. Halpern, Y et al., 'Using anchors to estimate clinical state without labeled data', AMIA Annual Symposium, Washington, DC USA, November 2014.

35. Halpern, $\mathrm{Y}$ et al., 'Electronic medical record phenotyping using the anchor and learn framework', $J$ AMIA, Vol. 23, 2016.

36. Lipton, Z, 'The mythos of model interpretability', ICML, New York, NY USA, June 2016.

37. Wu, $\mathrm{M}$ et al., 'Beyond sparsity: Tree regularization of deep models for interpretability', AAAI, New Orleans, LA USA, February 2018.

38. Glass, K and R Colbaugh, 'Interpretable prediction models for EHRs', Technical Report, Volv Global, Lausanne, Switzerland, February 2019.

39. O’Malley, K et al., 'Measuring diagnoses: ICD code accuracy', Health Services Research, Vol. 40, 2005.

40. Wells, B et al., 'Strategies for handling missing data in electronic health record derived data', eGEMs, Vol. 1, 2013.

41. Kamkar, I et al., 'Stable feature selection for clinical prediction: Exploiting ICD tree structure using TreeLASSO', J Biomedical Informatics, Vol. 53, 2015.

42. Giunchiglia, F and T Walsh, ‘A theory of abstraction’, Artificial Intelligence, Vol. 57, 1992.

43. Colbaugh, R and K Glass, 'Anticipating complex network vulnerabilities through abstraction-based analysis', Security Informatics, Vol. 1, 2012.

44. Stears, A and C Hames, 'Diagnosis and management of lipodystrophy: A practical update', Clinical Lipidology, Vol. 9, 2014. 